\title{
Errata: The Effects of Environment and Aggregation on the Absorption Spectra of Dyes
}

\author{
[Rev. Mod. Phys. 14, 303 (1942)] \\ S. E. SHEPPARD \\ Research Laboratories, Eastman Kodak Company, Rochester, New York
}

\begin{abstract}
THERE are several errata to the above paper which was published in the April-July issue of the Reviews of Modern Physics. They are as follows:
\end{abstract}

1. On p. 304, under the paragraph headed "Solutions," line 3, the expression for band strength should be $\int \epsilon d v$.

2. On p. 306, the caption to Fig. 5 should read: Variation of absorption with refractive index. . . .

3. Also on p. 306, the caption to Fig. 6 should read: Variation of absorption of merocyanine in polar solvents. (1) aliphatic series, (2) aromatic series, (3) amines.

Note there should be no "refractive index" and (3) is "amines" and not "amino."

4. On p. 309, in the left-hand column of text, the diagram and the paragraph set in small type should have been included in footnote 2 at the bottom of that page. Note footnote 2 now has nothing following the colon.

5. On p. 311, in the left-hand column of text, the word "Kekule" (first word in the line) should be enclosed in parentheses.

6. On p. 331 , in the structural formulas for diethyl- $\psi$-cyanine and diethyl- $\psi$ selena-cyanine, there should be a double bond connecting the positively charged nitrogen with the carbon next to the methine linkage:<smiles>CCN=C1CCC2CCCCC2N=C1C=C1CCC2CCCCC2N1CC</smiles>

diethyl- $\psi$-cyanine

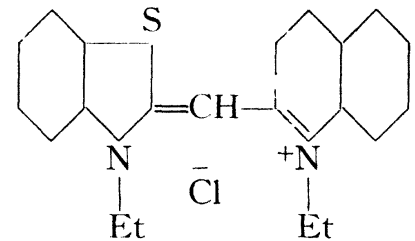

and

diethyl- $\psi$-selena-cyanine 